\title{
Subcycle contact-free nanoscopy of ultrafast interlayer transport in atomically thin heterostructures
}

DOI:

10.1038/s41566-021-00813-y

\section{Document Version}

Accepted author manuscript

Link to publication record in Manchester Research Explorer

\section{Citation for published version (APA):}

Plankl, M., Faria Junior, P. E., Mooshammer, F., Siday, T., Zizlsperger, M., Sandner, F., Schiegl, F., Maier, S., Huber, M. A., Gmitra, M., Fabian, J., Boland, J. L., Cocker, T. L., \& Huber, R. (2021). Subcycle contact-free nanoscopy of ultrafast interlayer transport in atomically thin heterostructures. Nat Photon, 15(8), 594-600. https://doi.org/10.1038/s41566-021-00813-y

Published in:

Nat Photon

\section{Citing this paper}

Please note that where the full-text provided on Manchester Research Explorer is the Author Accepted Manuscript or Proof version this may differ from the final Published version. If citing, it is advised that you check and use the publisher's definitive version.

\section{General rights}

Copyright and moral rights for the publications made accessible in the Research Explorer are retained by the authors and/or other copyright owners and it is a condition of accessing publications that users recognise and abide by the legal requirements associated with these rights.

\section{Takedown policy}

If you believe that this document breaches copyright please refer to the University of Manchester's Takedown Procedures [http://man.ac.uk/04Y6Bo] or contact uml.scholarlycommunications@manchester.ac.uk providing relevant details, so we can investigate your claim.

\section{OPEN ACCESS}




\section{Subcycle contact-free nanoscopy of ultrafast interlayer transport in atomically thin heterostructures}

M. Plank1 ${ }^{1}$, P. E. Faria Junior ${ }^{1}$, F. Mooshammer ${ }^{1}$, T. Siday ${ }^{1}$, M. Zizlsperger ${ }^{1}$, F. Sandner ${ }^{1}$, F. Schieg1 ${ }^{1}$, S. Maier ${ }^{1}$, M. A. Huber ${ }^{1,4}$, M. Gmitra ${ }^{1,5}$, J. Fabian ${ }^{1, *}$, J. L. Boland ${ }^{1,2}$, T. L. Cocker ${ }^{1,3}$ and R. Huber ${ }^{1, *}$ ${ }^{I}$ Department of Physics and Regensburg Center for Ultrafast Nanoscopy (RUN), University of Regensburg, 93053 Regensburg, Germany

${ }^{2}$ Photon Science Institute, Department of Electrical and Electronic Engineering, University of Manchester, Manchester M13 9PL, UK

${ }^{3}$ Department of Physics and Astronomy, Michigan State University, East Lansing, MI 48824, USA ${ }^{4}$ Department of Applied Physics, Stanford University, Stanford, CA 94305, USA, and Stanford PULSE Institute, SLAC National Accelerator Laboratory, Menlo Park, CA 94025, USA ${ }^{5}$ Present address: Institute of Physics, Pavol Jozef Šafárik University in Košice, 04001 Košice, Slovakia. *email: jaroslav.fabian@ur.de,rupert.huber@ur.de

Tunnelling is one of the most fundamental manifestations of quantum mechanics. The recent advent of lightwave-driven scanning tunnelling microscopy has revolutionized ultrafast nanoscience by directly resolving electron tunnelling in electrically conducting samples on the relevant ultrashort length and time scales. Here, we introduce a complementary approach based on terahertz near-field microscopy to perform ultrafast nano-videography of tunnelling processes even in insulators. The central idea is to probe the evolution of the local polarizability of electron-hole pairs with evanescent terahertz fields, which we detect with subcycle temporal resolution. In a concept proof, we resolve femtosecond interlayer transport in van der Waals heterobilayers and reveal pronounced variations of the local formation and annihilation of interlayer excitons on deeply subwavelength, nanometre scales. Such contact-free nanoscopy of tunnelling-induced dynamics should be universally applicable to conducting and non-conducting samples and reveal how ultrafast transport processes shape functionalities in a wide range of condensed matter systems. 
Ultrafast scanning tunnelling microscopy ${ }^{1-10}$ (STM) has turned a long-standing dream into reality: femtosecond snapshot imaging on the atomic scale is now possible ${ }^{2,4,8}$. By biasing the tip-sample junction of an STM with the oscillating carrier field of light, one can reach a time resolution faster than a cycle of light, and access the motion of a single molecule ${ }^{2,8}$. Yet, the concept requires electrically conducting samples and the electrical current in the STM junction may perturb intrinsic tunnelling processes within the sample. Tunnelling microscopy on insulators has recently become accessible with a new tip-tapping AC STM concept ${ }^{11}$, but it does not provide ultrafast temporal resolution. Often, the best of both worlds - access to insulating samples and sensitivity to tunnelling with time resolution faster than an oscillation cycle of light - is required.

Atomically thin heterostructures of van der Waals monolayers represent a prototypical example in which the optoelectronic properties sensitively depend on ultrafast electron tunnelling. For example, interlayer hopping is at the heart of superconducting ${ }^{12}$ and Mott ${ }^{13,14}$ transitions, ultrafast photodetectors ${ }^{15}$ and nanoscale magnetoresistance devices ${ }^{16,17}$. In transition metal dichalcogenide (TMDC) heterobilayers $^{18}$, type-II band alignment enables electron tunnelling ${ }^{19-21}$, which is crucial for atomically thin $\mathrm{p}-\mathrm{n}$ junctions $\mathrm{s}^{22}$, solar cells $\mathrm{s}^{23}$, and the formation of interlayer excitons ${ }^{19,24}$. These spatially separated, bound electron-hole $(e-h)$ pairs hold great promise for high-temperature condensation and future information technologies ${ }^{21}$. A microscopic understanding of interlayer charge transfer and recombination in van der Waals (vdW) heterostructures calls for non-invasive nanoscopy of ultrafast tunnelling processes.

Running an external current through the layered structure risks altering the intrinsic ultrafast interlayer dynamics. Meanwhile, diffraction-limited optical probes average over the nanoscale inhomogeneities of strain, doping, or dielectric disorder ${ }^{25}$. Conversely, near-field microscopy has accessed the ultrafast linear ${ }^{26-28}$ and nonlinear ${ }^{29}$ optical responses of vdW systems with spatial resolution many orders of magnitude below the diffraction limit. In this way, intersubband transitions in few-layer TMDCs ${ }^{30}$ and the impact of domain boundaries or moiré superlattices on surface polariton propagation have been revealed ${ }^{31}$. However, spatiotemporal studies of the unique out-of-plane tunnelling and decay dynamics in vdW heterostructures have thus far remained out of reach. 
Here, we use ultrafast scattering-type scanning near-field optical microscopy ${ }^{29,32,33}$ (s-SNOM) at terahertz $(\mathrm{THz})$ frequencies ${ }^{27,34-43}$ as a non-invasive, subcycle, and contact-free probe of charge transfer dynamics in $\mathrm{WSe}_{2} / \mathrm{WS}_{2}$ heterostructures on insulating substrates. Our approach builds on a critical change of the polarizability of $e-h$ pairs during interlayer tunnelling, as explained by ab initio density functional theory and confirmed by $\mathrm{THz}$ emission directly linked to the ultrafast charge separation. We track the subcycle polarizability with $\mathrm{THz}$ near fields confined to the tip apex to map the nanoscale build-up and decay of interlayer $e-h$ pair populations.

\section{Results}

Subcycle non-invasive polarization nanoscopy of ultrafast charge separation. The experimental principle is sketched in Fig. 1a. Femtosecond light pulses (centre wavelength, $515 \mathrm{~nm}$; pulse duration, $140 \mathrm{fs}$ ) photo-generate $e-h$ pairs by interband transitions within each of the monolayers of a $\mathrm{WSe}_{2} / \mathrm{WS}_{2}$ heterostructure prepared by mechanical exfoliation and all-dry viscoelastic stamping (see Methods). Type-II band alignment (Fig. 1a, inset) then allows electrons and holes to separate into the $\mathrm{WS}_{2}$ and the $\mathrm{WSe}_{2}$ layer, respectively, by interlayer tunnelling. To record this ultrafast process on the nanoscale, we probe the polarizability of the $e-h$ pairs with the near field of a single-cycle THz pulse coupled to the nanoscale tip of an s-SNOM (Fig. 1a, red arrows). Qualitatively, $e-h$ pairs that are quantum confined into a single monolayer should screen external fields less efficiently than pairs delocalized over two monolayers. Such a difference in polarizability may leave a measurable fingerprint in the scattered THz field $E^{\text {scat}}$, which we detect by electro-optic sampling ${ }^{33,38,43}$ (see Methods).

The probe frequency is chosen to remain below all relevant electronic resonances, such that the polarization of the $e-h$ pairs can follow the external field quasi-instantaneously. For background suppression, we demodulate the electric fields $E_{\mathrm{n}}$ at the $\mathrm{n}^{\text {th }}$ harmonic of the tip tapping frequency. Excellent sensitivity is required because of the extremely sub-wavelength dimensions of the light-matter interaction region. Its volume, set by the tip radius of curvature $(<20 \mathrm{~nm})$ and the thickness of the heterostructure $(\sim 1 \mathrm{~nm})$, is smaller than the diffraction limit of $\mathrm{THz}$ radiation by more than 12 orders of magnitude. To observe clear nanoscopic pump-probe signals, we pioneer a new combination of ultrafast nanoscopy with a high-power, ultrastable Yb:YAG thin-disc laser source operating at high repetition rates (see Methods and Supplementary Section 1). 
In Fig. 1 b, a representative scattered waveform $E_{1}^{\text {scat }}$ (black) recorded on the equilibrium

$\mathrm{WSe}_{2} / \mathrm{WS}_{2}$ heterostructure is depicted together with the maximum differential change $\Delta E_{1}^{\text {scat }}$ (blue) induced by the visible pump pulse. Interestingly, $\Delta E_{1}^{\text {scat }}$ displays a phase shift of $90^{\circ}$ with respect to $E_{1}^{\text {scat }}$, whereas the overall temporal shape of the electric field is mostly preserved. The same holds true for $\Delta E_{1}^{\text {scat }}$ obtained on a $\mathrm{WSe}_{2}$ monolayer (orange). Thus, the relative photo-induced amplitude and phase $\left(\Delta E_{1}^{\text {scat }} / E_{1}^{\text {scat }}\right)$ exhibit no distinct signatures within our spectral range (Fig. 1c). This is expected, as even low-energy electronic quantum transitions such as Lyman-like $1 s-2 p$ resonances of excitons lie more than an order of magnitude above our probing frequency ${ }^{19}$. Remarkably, the pump-induced signal from the heterobilayer is substantially larger than twice the monolayer response. We will show below that this observation is well explained by the dramatic increase of the non-resonant subcycle polarization of $e-h$ pairs $^{44}$ upon tunnelling into different monolayers.

To illuminate the ultrafast photo-carrier dynamics, we trace the peak of the photo-induced waveform, $\Delta \hat{E}_{1}^{\text {scat }}$, which yields the spectrally integrated response as a function of the pump delay time $t_{\mathrm{p}}$. Interestingly, there are significant differences in the ultrafast charge carrier dynamics in monolayer and heterostructure areas (Fig. 1d,e). The pump-probe traces of the monolayers exhibit a rapid onset within the response time of our setup (see Methods and Supplementary Section 1) as unbound $e-h$ pairs are injected and intralayer excitons are expected to form ${ }^{45}$. The subsequent decay of $\Delta \hat{E}_{1}^{\text {scat }}$ within $\sim 2 \mathrm{ps}$ is consistent with spatially averaged lifetimes determined in far-field experiments ${ }^{19}$. In contrast, the heterostructure behaves profoundly differently: the magnitude of $\Delta \hat{E}_{1}^{\text {scat }}$ is increased by up to a factor of 8 and the decay is drastically slowed down to $\sim 100$ ps (Fig. 1e, inset), indicating the formation of long-lived interlayer excitons. Moreover, the maximum of the pump-probe trace is significantly delayed (vertical dashed lines). These signatures reflect the interlayer tunnelling and subsequent recombination of highly polarizable, spatially separated $e-h$ pairs, as we demonstrate next.

Polarizabilities of intra- and interlayer electron-hole pairs. We first show that $e-h$ pairs located in mono- and bilayers exhibit dramatically different responses to a quasi-static, dominantly out-of-plane THz electric field (Fig. 2a). Our ab initio density functional theory directly provides the band structures and the underlying wavefunctions, including spin-orbit coupling (see Methods and Supplementary 
Section 2). For both the unbiased $\mathrm{WS}_{2}$ monolayer (Fig. 2b, left) and the unbiased $\mathrm{WSe}_{2} / \mathrm{WS}_{2}$ heterobilayer (Fig. 2c, left), the orbital structure of the relevant valence and conduction band states (which host photo-induced holes and electrons, respectively) is localized around the transition metal atoms. An out-of-plane electric field redistributes the photo-induced charge density differently in the two cases: while the charge redistribution in the monolayer is almost mirror-symmetric along the outof-plane direction (Fig. 2b, right), the heterobilayer exhibits a clear asymmetry and the charge is deflected even beyond the chalcogen atoms in $\mathrm{WSe}_{2}$ (Fig. 2c, right). By evaluating the quadratic component of the field-induced energy shift, we determine the out-of-plane polarizabilities $\alpha_{\perp}$ of the $e-$ $h$ pairs (Fig. 2d, Supplementary Section 2). Electrons confined into a single monolayer are only weakly susceptible to out-of-plane electric fields ${ }^{46}$, whereas the finite extension of the wavefunction in heterostructures drastically increases the deflection of wave packets by an external stimulus by up to one order of magnitude.

Our experimental sensitivity to in-plane components of the THz near field is smaller than to its outof-plane counterpart by more than two orders of magnitude (Supplementary Section 3). Yet, the in-plane polarizability of monolayer materials has been reported to exceed typical values of the out-of-plane polarizability by one to two orders of magnitude (see Supplementary Section 4), at least partially compensating for the relative insensitivity to in-plane fields. Nonetheless, the in-plane polarizability $\alpha_{\|}$ is larger for interlayer $e-h$ pairs than for intralayer pairs by approximately the same factor as for out-ofplane polarizabilities $\alpha_{\perp}$. To illustrate this fact, we compute two-dimensional, atom-like ${ }^{47}$ excitonic wavefunctions with established dielectric screening and exciton Bohr radii ${ }^{19}$ and compare the effect of an in-plane electric field on intralayer (Fig. 2e) and interlayer excitons (Fig. 2f). Spatial separation into different monolayers weakens the Coulomb attraction between electron and hole, increasing $\alpha_{\|}$by one order of magnitude, as also confirmed by recent experiments resolving the mid-infrared dielectric functions $^{19}$ (Fig. 2g, Supplementary Section 5). Thus, irrespective of the probe field direction, the tunnelling process transforming photoexcited intralayer $e-h$ pairs into interlayer species can be followed by resolving the dramatic change in polarizability during the charge transfer process. Moreover, the ratios between the experimental values for $\Delta \hat{E}_{1}^{\text {scat }}$ recorded on the monolayers and the heterostructure in Fig. 1e are reproduced well by the theoretically predicted polarizabilities (Fig. 2d,g). 
we compare the delayed onset of the pump-probe signal from the heterostructure with the tunnelling dynamics independently measured by laser $\mathrm{THz}$ emission nanoscopy ${ }^{38,41,43}$ (LTEN). Interlayer tunnelling should generate a time-dependent out-of-plane current of density $j_{z}(t)$ that produces coherent $\mathrm{THz}$ emission ${ }^{20}$, providing direct access to the ultrafast charge transfer dynamics. This emission is scattered into the far field for electro-optic detection ${ }^{38,43}$ (Fig. 3a). The ultimately thin sample requires us to push this technique to record sensitivity. After sufficient averaging (more than one hour), a clean $\mathrm{THz}$ waveform $E_{1}^{\mathrm{em}}$ consisting of an asymmetric single cycle with a period of $\sim 1 \mathrm{ps}$ emerges from a photoexcited $\mathrm{WSe}_{2} / \mathrm{WS}_{2}$ heterobilayer (Fig. 3b, blue). The field polarity and hence the direction of the out-of-plane current $j_{z}(t)$ flip when the stacking order of the monolayers is inverted (Fig. 3b, purple). Since no emission signatures are obtained from the substrate or the monolayer regions (Fig. 3b, grey), we unambiguously identify interlayer charge transfer as the origin of the detected waveforms.

From the LTEN temporal waveform, the local tunnelling current $j_{\mathrm{z}}(t)$ can be reconstructed: we parameterize $j_{\mathrm{z}}(t)$ with an effective time constant $\tau_{\text {tunnel }}$ and numerically propagate the emitted $\mathrm{THz}$ transient from the tip apex to the electro-optic detector using finite element method simulations ${ }^{48}$ (see Methods, Supplementary Section 6 and 7, and Supplementary Movies 1 and 2). Our experimental emission waveforms are well reproduced for $\tau_{\text {tunnel }}=200$ fs (Fig. 3b, red) - a value that is compatible with previous experimental results ${ }^{19,20,49}$ and coincides with the response time of our setup. Most importantly, the temporal build-up of the density of spatially separated $e-h$ pairs (Fig. 3c, red curve) perfectly matches with the onset of the pump-probe signal from the heterostructure (Fig. 1e, red broken line, see also Supplementary Section 8). These observations validate our polarization nanoscopy as an ultrafast contact-free probe of tunnelling $e-h$ pairs that works by monitoring the population of the interlayer species.

Contact-free subcycle nanovideography. While LTEN can quantify interlayer tunnelling on the nanoscale, it is insensitive to the dynamics following the tunnelling process and is limited by the low number of photons spontaneously emitted by the accelerated $e-h$ pairs. Conversely, the signal-to-noise ratio of polarization nanoscopy can be scaled by the incident coherent probe field. Our setup enables a 
polarization signal up to 25 times larger than the $\mathrm{THz}$ emission field, reducing the data acquisition time by almost three orders of magnitude and facilitating nanoscale images even if only $\sim 10-100$ charge carriers per laser pulse give rise to the detected signals. Most importantly, THz probing is independent of interband optical selection rules. Owing to the weak spatial overlap of electron and hole wavefunctions, interlayer excitons are optically dark in interband absorption ${ }^{19}$. Even photoluminescence (PL) from interlayer excitons is typically so weak that micro-PL measurements cannot be directly traced to a local density distribution of interlayer $e-h$ pairs. Our new polarization nanoscopy not only directly probes local $e-h$ pair densities, but also adds the essential benefits of nanometre spatial resolution and ultrafast temporal resolution, far outperforming micro-PL.

To illustrate how this new approach reveals previously elusive ultrafast and spatially inhomogeneous dynamics of optically dark $e-h$ pairs, we record femtosecond snapshots of a representative sample region for different delay times $t_{\mathrm{p}}$ after photoexcitation. At each point in space, we analyse the peak of the pump-induced change to the electric field $\Delta \hat{E}_{1}^{\text {scat }}$ as a function of $t_{\mathrm{p}}$ and fit the respective trace with a mono-exponential decay of the form $\Delta E_{1}^{\max } \cdot e^{-t / \tau}$ (Fig. 4a inset and Supplementary Movie 3). The amplitude $\Delta E_{1}^{\max }$ and the decay constant $\tau$ are measures of the local density and the lifetime of all interlayer $e-h$ pairs (i.e. including optically dark pairs), respectively. Figures $4 \mathrm{a}$ and $4 \mathrm{~b}$ show the first nanoscale measurement of these quantities as colour-coded maps on the three-dimensional topography of the heterostructure. Subtle variations in $\Delta E_{1}^{\max }$ (Fig. 4a) indicate that the formation of interlayer $e-h$ pairs may be modulated by previously undetected nanoscale differences in charge transfer efficiency. The lifetime $\tau$ (Fig. 4b) of the spatially separated $e-h$ pairs varies by as much as a factor of three within the investigated area. Local regions of extended lifetimes seem to correlate with high photoinduced pair densities (Supplementary Section 9). Conversely, negligible densities and short lifetimes are observed at topographic irregularities, which we attribute to spatial separation of the heterostructure into its constituent monolayers. Remarkably, we observe significant differences in $e-h$ pair lifetimes between topographically similar flat regions (Fig. 4a, inset).

Figure $4 \mathrm{c}$ displays close-up images of the flat regions indicated in Fig. $4 \mathrm{~b}$ with spatial resolution better than $50 \mathrm{~nm}$ (Supplementary Section 10). Intriguingly, while the magnitude of $\Delta \hat{E}_{1}^{\text {scat }}$ (lower 
panels) varies slowly within these regions, it appears immune to few-nanometre-tall topographic folds

193 (top panels). We therefore conclude that the heterostructure remains intact on the nanoscale, with the interlayer exciton likely robust to the subtle changes to the local dielectric environment. Hence, we propose that the larger area of extended lifetime shown in Figure $4 \mathrm{~b}$ (blue square) could arise from an advantageous local atomic registry, through twist angle ${ }^{19}$ or strain ${ }^{50}$. Such hitherto inaccessible local information is essential for the optimization of $\mathrm{vdW}$ heterostructures and foreshadows the power of using ultrafast THz s-SNOM as a contact-free probe of tunnelling.

\section{Discussion}

In conclusion, we have exploited the transient polarizability of electron-hole pairs for non-invasive THz nanoscopy of ultrafast interlayer transport in atomically thin heterostructures. Our experiments not only make use of the versatile portfolio of ultrafast THz near-field microscopy, including LTEN and sSNOM, but also conceptually bridge the gap to lightwave-driven STM. In contrast to ultrafast STM, though, our approach offers spatiotemporal access to tunnelling processes without driving external currents - a concept that should readily lend itself to the observation of tunnelling in insulating, conducting, or superconducting systems on subcycle time scales. Already, we have demonstrated that the interlayer tunnelling processes that govern phase transitions, energy harvesting and light-emission in atomically thin heterostructures are now accessible in situ on the relevant length and time scales. Looking forward, this may also facilitate the visualization of photo-carrier trapping in moiré potentials. 


\section{References}

211 1. Cocker, T. L. et al. An ultrafast terahertz scanning tunnelling microscope. Nat. Photonics 7, $212 \quad 620-625(2013)$.

213 2. Cocker, T. L., Peller, D., Yu, P., Repp, J. \& Huber, R. Tracking the ultrafast motion of a single molecule by femtosecond orbital imaging. Nature 539, 263-267 (2016).

3. Yoshioka, K. et al. Real-space coherent manipulation of electrons in a single tunnel junction by single-cycle terahertz electric fields. Nat. Photonics 10, 762-765 (2016).

4. Jelic, V. et al. Ultrafast terahertz control of extreme tunnel currents through single atoms on a silicon surface. Nat. Phys. 13, 591-598 (2017).

5. Yoshioka, K. et al. Tailoring Single-Cycle Near Field in a Tunnel Junction with CarrierEnvelope Phase-Controlled Terahertz Electric Fields. Nano Lett. 18, 5198-5204 (2018).

6. Yoshida, S. et al. Subcycle Transient Scanning Tunneling Spectroscopy with Visualization of Enhanced Terahertz Near Field. ACS Photonics 6, 1356-1364 (2019).

7. Garg, M. \& Kern, K. Attosecond coherent manipulation of electrons in tunneling microscopy. Science 367, 411-415 (2020).

8. Peller, D. et al. Sub-cycle atomic-scale forces coherently control a single-molecule switch. Nature 585, 58-62 (2020).

9. Müller, M., Martín Sabanés, N., Kampfrath, T. \& Wolf, M. Phase-Resolved Detection of Ultrabroadband THz Pulses inside a Scanning Tunneling Microscope Junction. ACS Photonics 7, 2046-2055 (2020).

10. Peller, D. et al. Quantitative sampling of atomic-scale electromagnetic waveforms. Nat. Photonics 15, 143-147 (2021).

11. Patera, L. L., Queck, F., Scheuerer, P. \& Repp, J. Mapping orbital changes upon electron transfer with tunnelling microscopy on insulators. Nature 566, 245-248 (2019). 556, $43-50(2018)$. 
13. Tang, Y. et al. Simulation of Hubbard model physics in $\mathrm{WSe}_{2} / \mathrm{WS}_{2}$ moiré superlattices. Nature 579, 353-358 (2020).

14. Balents, L., Dean, C. R., Efetov, D. K. \& Young, A. F. Superconductivity and strong correlations in moiré flat bands. Nat. Phys. 16, 725-733 (2020).

15. Massicotte, M. et al. Picosecond photoresponse in van der Waals heterostructures. Nat. Nanotechnol. 11, 42-46 (2016).

16. Klein, D. R. et al. Probing magnetism in 2D van der Waals crystalline insulators via electron tunneling. Science 360, 1218-1222 (2018).

17. Song, T. et al. Giant tunneling magnetoresistance in spin-filter van der Waals heterostructures. Science 360, 1214-1218 (2018).

18. Novoselov, K. S., Mishchenko, A., Carvalho, A. \& Castro Neto, A. H. 2D materials and van der Waals heterostructures. Science 353, aac9439 (2016).

19. Merkl, P. et al. Ultrafast transition between exciton phases in van der Waals heterostructures. Nat. Mater. 18, 691-696 (2019).

20. Ma, E. Y. et al. Recording interfacial currents on the subnanometer length and femtosecond time scale by terahertz emission. Sci. Adv. 5, eaau0073 (2019).

21. Jauregui, L. A. et al. Electrical control of interlayer exciton dynamics in atomically thin heterostructures. Science 366, 870-875 (2019).

22. Lee, C.-H. et al. Atomically thin p-n junctions with van der Waals heterointerfaces. Nat. Nanotechnol. 9, 1-29 (2014).

23. Furchi, M. M., Pospischil, A., Libisch, F., Burgdörfer, J. \& Mueller, T. Photovoltaic effect in an electrically tunable Van der Waals heterojunction. Nano Lett. 14, 4785-4791 (2014).

24. Rivera, P. et al. Interlayer valley excitons in heterobilayers of transition metal dichalcogenides. Nat. Nanotechnol. 13, 1004-1015 (2018).

25. Raja, A. et al. Dielectric disorder in two-dimensional materials. Nat. Nanotechnol. 14, 832-837 (2019). 
26. Park, K.-D., Jiang, T., Clark, G., Xu, X. \& Raschke, M. B. Radiative control of dark excitons at room temperature by nano-optical antenna-tip Purcell effect. Nat. Nanotechnol. 13, 59-64 (2018).

27. Zhang, J. et al. Terahertz Nanoimaging of Graphene. ACS Photonics 5, 2645-2651 (2018).

28. Bao, W. et al. Visualizing nanoscale excitonic relaxation properties of disordered edges and grain boundaries in monolayer molybdenum disulfide. Nat. Commun. 6, 7993 (2015).

29. Jiang, T., Kravtsov, V., Tokman, M., Belyanin, A. \& Raschke, M. B. Ultrafast coherent nonlinear nanooptics and nanoimaging of graphene. Nat. Nanotechnol. 14, 838-843 (2019).

30. Schmidt, P. et al. Nano-imaging of intersubband transitions in van der Waals quantum wells. Nat. Nanotechnol. 13, 1035-1041 (2018).

31. Sunku, S. S. et al. Photonic crystals for nano-light in moiré graphene superlattices. Science 362, 1153-1156 (2018).

32. Huber, M. A. et al. Femtosecond photo-switching of interface polaritons in black phosphorus heterostructures. Nat. Nanotechnol. 12, 207-211 (2017).

33. Eisele, M. et al. Ultrafast multi-terahertz nano-spectroscopy with sub-cycle temporal resolution. Nat. Photonics 8, 841-845 (2014).

34. Huber, A. J., Keilmann, F., Wittborn, J., Aizpurua, J. \& Hillenbrand, R. Terahertz Near-Field Nanoscopy of Mobile Carriers in Single Semiconductor Nanodevices. Nano Lett. 8, 3766-3770 (2008).

35. Siday, T., Hale, L. L., Hermans, R. I. \& Mitrofanov, O. Resonance-Enhanced Terahertz Nanoscopy Probes. ACS Photonics 7, 596-601 (2020).

36. Moon, K. et al. Subsurface Nanoimaging by Broadband Terahertz Pulse Near-Field Microscopy. Nano Lett. 15, 549-552 (2015).

37. Kuschewski, F. et al. Narrow-band near-field nanoscopy in the spectral range from 1.3 to 8.5 THz. Appl. Phys. Lett. 108, 113102 (2016).

38. Klarskov, P., Kim, H., Colvin, V. L. \& Mittleman, D. M. Nanoscale Laser Terahertz Emission 
Microscopy. ACS Photonics 4, 2676-2680 (2017).

289

290

291

292

293

294

295

296

297

298

299

300

301

302

303

304

305

306

307

308

309

310

311

312

39. Stinson, H. T. et al. Imaging the nanoscale phase separation in vanadium dioxide thin films at terahertz frequencies. Nat. Commun. 9, 3604 (2018).

40. Mastel, S. et al. Terahertz Nanofocusing with Cantilevered Terahertz-Resonant Antenna Tips. Nano Lett. 17, 6526-6533 (2017).

41. Yao, Z. et al. Photo-induced terahertz near-field dynamics of graphene/InAs heterostructures. Opt. Express 27, 13611 (2019).

42. Chen, X. et al. Modern Scattering-Type Scanning Near-Field Optical Microscopy for Advanced Material Research. Adv. Mater. 31, 1804774 (2019).

43. Pizzuto, A., Mittleman, D. M. \& Klarskov, P. Laser THz emission nanoscopy and THz nanoscopy. Opt. Express 28, 18778 (2020).

44. Wang, F. et al. Exciton polarizability in semiconductor nanocrystals. Nat. Mater. 5, 861-864 (2006).

45. Steinleitner, P. et al. Direct Observation of Ultrafast Exciton Formation in a Monolayer of WSe 2 . Nano Lett. 17, 1455-1460 (2017).

46. Tian, T. et al. Electronic Polarizability as the Fundamental Variable in the Dielectric Properties of Two-Dimensional Materials. Nano Lett. 20, 841-851 (2020).

47. Yang, X. L., Guo, S. H., Chan, F. T., Wong, K. W. \& Ching, W. Y. Analytic solution of a twodimensional hydrogen atom. I. Nonrelativistic theory. Phys. Rev. A 43, 1186-1196 (1991).

48. Mooshammer, F. et al. Quantifying Nanoscale Electromagnetic Fields in Near-Field Microscopy by Fourier Demodulation Analysis. ACS Photonics 7, 344-351 (2020).

49. Hong, X. et al. Ultrafast charge transfer in atomically thin $\mathrm{MoS}_{2} / \mathrm{WS}_{2}$ heterostructures. Nat. Nanotechnol. 9, 1-5 (2014).

50. Bai, Y. et al. Excitons in strain-induced one-dimensional moiré potentials at transition metal dichalcogenide heterojunctions. Nat. Mater. 19, 1068-1073 (2020). 

ultrafast optical-pump/THz-probe s-SNOM experiments. The photo-generated $e-h$ pairs (blue and red spheres) are interrogated by a THz probe pulse $E_{\mathrm{THz}}$ as a function of the pump-probe delay time $t_{\mathrm{p}}$. The dominantly out-of-plane polarized THz fields beneath the tip apex (red arrows, see Supplementary Section 3 for distributions of the electric field) locally displace the $e-h$ pairs, such that their polarizability is encoded in the scattered fields $E^{\text {scat }}$. Inset: Schematic of the type-II band alignment of the heterostructure and the tunnelling of charge carriers through the van der Waals gap. b, Steadystate $\left(E_{1}^{\text {scat }}\right.$, black $)$ and pump-induced $\left(\Delta E_{1}^{\text {scat }}\right) \mathrm{THz}$ waveforms scattered off a $\mathrm{WSe}_{2} / \mathrm{WS}_{2}$ heterostructure (HS, blue; $t_{\mathrm{p}}=1.25 \mathrm{ps}$ ) and a $\mathrm{WSe}_{2}$ monolayer (ML, orange; $\left.t_{\mathrm{p}}=0.55 \mathrm{ps}\right)$. c, Relative photo-induced change to amplitude $\left(\Delta \tilde{E}_{1}^{\text {scat }} / \tilde{E}_{1}^{\text {scat }}\right.$, top panel) and phase spectra $\left(\Delta \phi_{1}-\phi_{1}\right.$, lower panel $)$ recorded on the heterostructure and the $\mathrm{WSe}_{2}$ monolayer. d, Atomic force microscopy image of a typical region featuring a $\mathrm{WS}_{2}$ monolayer (green, left) and heterostructure area (blue, right). e, Pumpinduced changes of the peak electric field $\Delta \hat{E}_{1}^{\text {scat }}$ for both monolayers (orange, green) and the heterostructure (blue) as a function of the pump delay time $t_{\mathrm{p}}$. The density of tunnelled $e-h$ pairs, corresponding to the build-up of the dipole moment (red, compare to Fig. 3c), matches the temporal evolution of $\Delta \hat{E}_{1}^{\text {scat }}$ of the HS. Inset: Additional data plotted for a larger range of delay times $t_{\mathrm{p}}$ recorded at the marked positions in $\mathbf{d}$. 
Figure 2 | Out-of-plane and in-plane polarizability of intralayer and interlayer electron-hole pairs.

332 a, Sketch of the electric-field-induced displacement of intra- and interlayer $e-h$ pairs highlighting the

333 larger susceptibility of interlayer excitons to out-of-plane electric fields. b, Charge density $\rho\left(E_{\mathrm{THz}}=0\right)$

$334=e \cdot\left(\left|\Psi_{\mathrm{VB}}\right|^{2}-\left|\Psi_{\mathrm{CB}}\right|^{2}\right)$ of the relevant valence $(\mathrm{VB})$ and conduction band $(\mathrm{CB})$ at the $\mathrm{K}$ point of a $\mathrm{WS}_{2}$

335 monolayer calculated by density functional theory in units of the elementary charge $e$ (left side). Photo-

336 induced mobile holes (electrons) contributing to the polarizability are located in the valence

337 (conduction) band and hence are labelled as positive (negative) charge density. On the right side, the

338 redistribution of charge density $\Delta \rho=\rho\left(E_{\mathrm{THz}}=1 \mathrm{~V} / \mathrm{nm}\right)-\rho\left(E_{\mathrm{THz}}=0\right)$ upon application of a quasi-static

339 out-of-plane electric field $E_{\mathrm{THz}}$ is visualized. For clarity, these data were calculated neglecting spin-orbit

340 coupling (see Supplementary Section 2). c, Charge density at the K point (left) and redistribution of

341 charge density (right) as in $\mathbf{b}$ of a lattice commensurate $\mathrm{WSe}_{2} / \mathrm{WS}_{2}$ heterostructure. The band extrema

342 of the valence and conduction bands relevant for interlayer $e-h$ pairs are localized in the top and bottom

343 layer, respectively. d, Out-of-plane polarizabilities $\alpha_{\perp}$ of the intra- and interlayer $e-h$ pairs in the

344 monolayer and heterostructure (with atomic relaxation) in c normalized to $\alpha_{\perp}$ of $\mathrm{WSe}_{2} / \mathrm{WS}_{2}$

345 (Supplementary Section 2). e, f, Modulus of the wavefunction $|\Psi(r)|$ of intralayer (e) and interlayer (f)

$3461 s$ A excitons for $E_{\mathrm{THz}}=0$ (left panels) and for an in-plane field $E_{\mathrm{THz}}=0.3 \mathrm{~V} / \mathrm{nm}$ (right panels) obtained

347 by analytically solving the two-dimensional hydrogen model ${ }^{47}$ with literature values ${ }^{19}$ (see

348 Supplementary Section 5). g, In-plane polarizabilities $\alpha_{\|}$of the intra- and interlayer $e-h$ pairs in

349 monolayers and heterostructure calculated with the model in e,f. For comparison, $\alpha_{\|}$extracted from the

350 dielectric function in ref. ${ }^{19}$ is also plotted (spheres). In each case, $\alpha_{\|}$is normalized to the respective $\alpha_{\|}$ 351 of $\mathrm{WSe}_{2} / \mathrm{WS}_{2}$. 
353 Figure 3 | Clocking ultrafast interlayer tunnelling using THz emission nanoscopy. a, Interlayer

354 transfer of electron-hole (e-h) pairs (blue and red spheres) in a $\mathrm{WSe}_{2} / \mathrm{WS}_{2}$ heterostructure causes an out-

355 of-plane current $j_{z}(t)$, which emits a characteristic THz transient. b, THz electric fields outcoupled by a 356 sharp metallic tip and electro-optically sampled in the far field, $E_{1}^{\mathrm{em}}$, as a function of the delay time.

$357 \mathrm{WSe} / \mathrm{WS}_{2}$ heterostructure: blue symbols; $\mathrm{WS}_{2} / \mathrm{WSe}_{2}$ heterostructure: purple symbols; $\mathrm{WS}_{2}$ monolayer:

358 grey symbols; dashed lines: guides to the eye; red line: simulated waveform based on the current density

359 depicted in $\mathbf{c}$, accounting for the tip transfer function and electro-optic detector response. The trailing

360 oscillations at $t=3 \mathrm{ps}$ are caused by reflection at the end of the cantilever (see Supplementary Section

3616 and 7, Supplementary Movies 1 and 2). Inset: amplitude spectra $\tilde{E}^{\mathrm{em}}$. c, Out-of-plane current density

$362 j_{z}$ (black) originating from interlayer charge transfer for a characteristic tunnelling time $\tau_{\text {tunnel }}=200 \mathrm{fs}$.

363 The tunnelled $e-h$ pairs (red) are obtained by numerical integration of the current density $j_{\mathrm{z}}(t)$. 

lifetimes by ultrafast contact-free nanoscopy. a,b, Magnitude of the pump-induced changes in scattered electric field $\Delta E_{1}^{\max }$ (a) and characteristic lifetime $\tau$ (b) of the photo-generated carriers colourcoded onto the topography of a representative region of the $\mathrm{WSe}_{2} / \mathrm{WS}_{2}$ heterostructure (recorded by atomic force microscopy). The two-dimensional maps were obtained by evaluating a series of s-SNOM

370 snapshot images and fitting a mono-exponential decay to the temporal evolution of $\Delta \widehat{E}_{1}^{\text {scat }}$ at each pixel

371 (for details see main text). For two representative, flat areas indicated by the arrows, the experimental

372 data (symbols) and fits (solid lines) are shown in the inset in a. c, Atomic force microscopy maps of the

373 flat areas (see coloured frames in $\mathbf{b}$, corner for orientation) and $\Delta \hat{E}_{1}^{\text {scat }}$ scans for different delay times.

374 The characteristic length scale of the modulation in $\Delta \hat{E}_{1}^{\text {scat }}(\sim 400 \mathrm{~nm})$ is significantly larger than both

375 the spatial resolution of our s-SNOM setup $(\sim 50 \mathrm{~nm})$ and the topographic features in the top panel. For

376 further details see Supplementary Section 10. Scale bar: $400 \mathrm{~nm}$. 


\section{Acknowledgements}

379

380

381

382

383

384

385

386

387

We thank Martin Furthmeier for technical assistance and Tony F. Heinz for fruitful discussions. This work was supported by the Deutsche Forschungsgemeinschaft (DFG, German Research Foundation)— Project-ID, 314695032-SFB 1277 (Subprojects A05 and B05) and through research grants HU1598/3 and CO1492/1. P.E.F.J. acknowledges funding by the A. v. Humboldt Foundation and by Capes (Grant No. 99999.000420/2016-06). M.A.H. was supported in part by the U.S. Department of Energy, Office of Science, Basic Energy Sciences (BES), Chemical Sciences, Geosciences, and Biosciences Division, AMOS Program. M.G. acknowledges support by the Ministry of Education, Science, Research and Sport of the Slovak Republic (Grand No. VEGA 1/0105/20). J.L.B. acknowledges support by the A. V. Humboldt Foundation and EPSRC (U.K.) via project EP/S037438/1.

\section{Author contributions}

M.P. and F.M. fabricated the samples. M.P., F.M., T.S., M.Z., F.Sa., F.Sc., S.M., M.A.H., J.L.B., T.L.C., and R.H. conducted the experiments. P.E.F.J., M.G. and J.F. performed the density functional theory calculations, F.M. performed the finite element simulations, and J.F, T.L.C. and R.H. supervised the study. M.P., F.M., and R.H. wrote the manuscript with input from all authors.

\section{Competing interests}

The authors declare no competing interests. 
Sample fabrication and characterization. The TMDC monolayers were fabricated via mechanical exfoliation $^{51}$. A thin flake of the material was exfoliated with Nitto tape (Nitto Denko, SPV 224P) from the bulk single crystal (bought at HQgraphene.com) and placed on a viscoelastic poly-dimethyl-siloxane (PDMS) film. By gently lifting the tape, a single monolayer of the TMDC was left on the PDMS film.

402

403

404 The monolayer was pre-characterised under an optical microscope before being stamped onto a silicon wafer covered with $300 \mathrm{~nm}$ of thermal oxide using a micro-positioning stage. This procedure was repeated to produce heterobilayers. The prepared heterostructures were annealed in high vacuum at $150^{\circ}$ Celsius for 2 hours to remove trapped adsorbates and to ensure good contact between the monolayers.

Ultrafast optical-pump/THz-probe near-field microscopy. The THz near-field microscopy experiments (Supplementary Fig. 1) are based on a high average-power, low-noise Yb:YAG thin-disc oscillator, which combines pulse energies of up to $3.75 \mu \mathrm{J}$ with a repetition rate of $24 \mathrm{MHz}$. These specifications yield the required sensitivity for retrieving the minute electric near fields of the emitted waveforms (LTEN) or for resolving the subtle changes to the scattered fields caused by the photoinduced polarizability (s-SNOM). The THz probe pulses are directly generated by optical rectification of 200-fs-long pulses centred at a wavelength of $1030 \mathrm{~nm}$ in a 2-mm-thick gallium phosphide (GaP) crystal. Sample photoexcitation is provided by the second harmonic (centre wavelength: $515 \mathrm{~nm}$; pulse duration: $140 \mathrm{fs}$ ) of the oscillator output generated using a $\beta$-barium borate crystal (thickness: $1.2 \mathrm{~mm}$ ). By modulating the pump beam using an acousto-optic modulator, background-free detection of the pump-induced change is achieved by serial lock-in detection. The THz and pump pulses are focused onto the tip of a commercial scattering-type scanning near-field optical microscope (NeaSNOM, Neaspec $\mathrm{GmbH}$ ) using a parabolic mirror with high numerical aperture. Subsequently, the residual pump radiation is separated from the scattered $\mathrm{THz}$ waveforms with a germanium wafer. The THz electric field is detected via electro-optic sampling ${ }^{33,36}$ in a second GaP crystal with a thickness of $4 \mathrm{~mm}$. The demodulated scattered field $E_{\mathrm{n}}$ is determined by analysing the harmonic of order $\mathrm{n}$ of the tip tapping frequency using a lock-in amplifier ${ }^{32,33,38,48,52}$. 
Based on the specifications described above, spectral components of the scattered electric fields can reliably be resolved up to a frequency of $\sim 2.5 \mathrm{THz}$. Consequently, we determine a response time of $\sim 400$ fs for our experimental setup. This value coincides with the quasi-instantaneous onset of the pumpprobe response of the monolayers in Fig. 1e.

Field polarity of the emitted waveforms. The polarity of the LTEN waveforms was calibrated to the underlying current using the photo-Dember effect on an indium arsenide (InAs) reference sample, where highly mobile electrons diffuse into the bulk material faster than the holes. This creates a net transient current $j_{\mathrm{z}}(t)$ towards the surface ${ }^{20,38}$, to which we assign a positive peak electric field. Since the transfer of electrons in the $\mathrm{WSe}_{2} / \mathrm{WS}_{2}$ heterostructure (Fig. 3a, purple arrow) corresponds to a current along the same out-of-plane direction as for InAs, the waveform (Fig. 3b, blue) has the same positive field polarity. Conversely, we find a negative peak electric field emitted from the $\mathrm{WS}_{2} / \mathrm{WSe}_{2}$ structure (Fig. 3b, purple), which confirms that the current is reversed in this case.

Finite element method simulation. The electromagnetic fields generated by the interlayer tunnelling current were calculated numerically using a commercial finite element method software (COMSOL Multiphysics, including the radiofrequency (RF) package). A spherical simulation volume with a diameter of $1.2 \mathrm{~mm}$ was chosen to accommodate the long wavelengths in the THz spectral range. In addition to these macroscopic dimensions, the nanoscale confinement of the electric field at the tip apex requires fine resolution, which renders realistic simulations of $\mathrm{THz}$ nanoscopy computationally demanding. Yet, a high-performance computer with 196 GB of RAM allows us to choose a fine mesh with up to one million individual elements. The exact geometry of tip and sample system is discussed in detail in Supplementary Section 6. The interlayer tunnelling current is modelled by a point-dipole source, which is placed $1 \mathrm{~nm}$ above the sample surface, at the centre of the simulation volume. For a range of frequencies up to $3 \mathrm{THz}$ (in steps of $0.1 \mathrm{THz}$ ), we then calculate the electromagnetic fields emerging from this source. Comparing the emitted far fields in the presence of the tip $E_{\mathrm{z}}^{\mathrm{far}, \text { tip }}$ with the ones emitted by the bare dipole $E_{\mathrm{z}}^{\text {far,bare }}$, the entire scattering response of the tip can be captured in a complex-valued transfer function $\Omega^{\text {transfer }}=E_{z}^{\text {far,tip }} / E_{z}^{\text {far,bare }}$ (see Supplementary Fig. 6c). 
For the emission field distributions, we superimpose the calculated frequency-domain field distributions according to the spectral weight set by the ultrafast temporal evolution of the interlayer tunnelling current in Fig. 1e and Fig. 3c. Here, we additionally account for the detector response of the electrooptic sampling (see Supplementary Section 7). By systematically evolving the phase of the complexvalued electric field for each frequency component individually, we obtain a time-domain movie of the emission process (see Supplementary Movies 1 and 2).

Numerical modelling of the interlayer current. We adapted a three-state mode $^{20}$, in which two main processes are expected to determine the interlayer current: (i) intralayer relaxation and (ii) interlayer transfer. Following the non-resonant photoexcitation $(2.4 \mathrm{eV})$, hot intralayer $e-h$ pairs most efficiently tunnel at the points of intersection of intra- and interlayer exciton dispersion relations, due to energy and momentum conservation ${ }^{19}$. This is accounted for by an additional delay at the rate $1 / \tau_{\text {relax }}$ before the carriers can tunnel into the adjacent layer at a rate $1 / \tau_{\text {tunnel }}$ and thereby contribute to the net interlayer current $j_{\mathrm{z}}(t)$. The current can then be written as $j_{\mathrm{z}}(t)=\left(\tau_{\text {tunnel }}-\right.$ $\left.\tau_{\text {relax }}\right)^{-1}\left[\exp \left(-\frac{t}{\tau_{\text {tunnel }}}\right)-\exp \left(-\frac{t}{\tau_{\text {relax }}}\right)\right]$. We found the best agreement with our experimental data by assuming $\tau_{\text {tunnel }} \approx \tau_{\text {relax }}$. In this case, both processes happen on similar time scales and cannot be distinguished in this experiment. This leads to a current of the form $j_{\mathrm{z}}(t)=\frac{t}{\tau_{\text {tunnel }^{2}}} \exp \left(-\frac{t}{\tau_{\text {tunnel }}}\right)$, with a characteristic duration of $\sim 2 \tau_{\text {tunnel }}$ (see Fig. 1e). Additionally, the current is convoluted with a Gaussian with a full width at half maximum of 140 fs to include the finite duration of the pump pulse. Finally, we use Maxwell's equations to calculate the emitted waveform based on $j_{z}(t)$, where we additionally account for the tip and detector transfer functions (for further details see Supplementary Section 7).

Ab initio DFT calculations. The electronic structures of monolayers $\mathrm{WS}_{2}, \mathrm{WSe}_{2}$ and their heterostructure were obtained via the full-potential linearized augmented plane-wave method as implemented in the Wien2k package ${ }^{53}$. The Perdew-Burke-Ernzerhof ${ }^{54}$ exchange-correlation functional was considered and, for the heterostructure, van der Waals interactions were accounted for using the D3 correction $^{55}$. The wavefunctions are expanded in atomic spheres with orbital quantum numbers up to 10 
475 and the plane-wave cut-off multiplied with the smallest atomic radii is set to 8. Spin-orbit coupling was

476 included fully relativistically for core electrons, while valence electrons were treated within a second-

477 variational procedure with the scalar-relativistic wavefunctions calculated in an energy window up to 5

478 Ry. Self-consistency was achieved using a Monkhorst-Pack k-grid of $15 \times 15 \times 1$ with convergence criteria

479 of $10^{-6} e$ for the charge and $10^{-6} \mathrm{Ry}$ for the energy. To account for the lattice mismatch in the

480 heterostructure, $\mathrm{WS}_{2}$ and $\mathrm{WSe}_{2}$ layers are strained by $1.81 \%$ and $-1.74 \%$, respectively. The optimized

481 interlayer distance with spin-orbit coupling is $3.6389 \AA$, consistent with recent calculations ${ }^{56}$. The

482 strained lattice parameters were taken from ref. ${ }^{57}$ and a vacuum space of $20 \AA$ was considered. The out-

483 of-plane electric field was modelled with a zig-zag potential added to exchange-correlation functional ${ }^{58}$

484 with 40 Fourier coefficients. For additional details, see Supplementary Section 2.

485

486 Data availability. The data sets generated during and/or analysed during the current study are

487 available from the corresponding authors upon reasonable request. 


\section{References}

51. Castellanos-Gomez, A. et al. Deterministic transfer of two-dimensional materials by all-dry viscoelastic stamping. 2D Mater. 1, 011002 (2014).

52. Ni, G. X. et al. Ultrafast optical switching of infrared plasmon polaritons in high-mobility graphene. Nat. Photonics 10, 244-247 (2016).

53. Blaha, P. et al. WIEN2k: An APW+lo program for calculating the properties of solids. $J$. Chem. Phys. 152, 074101 (2020).

54. Perdew, J. P., Burke, K. \& Ernzerhof, M. Generalized gradient approximation made simple. Phys. Rev. Lett. 77, 3865-3868 (1996).

55. Grimme, S., Antony, J., Ehrlich, S. \& Krieg, H. A consistent and accurate ab initio parametrization of density functional dispersion correction (DFT-D) for the 94 elements $\mathrm{H}-\mathrm{Pu}$. J. Chem. Phys. 132, 154104 (2010).

56. Yuan, L. et al. Twist-angle-dependent interlayer exciton diffusion in $\mathrm{WS}_{2}-\mathrm{WSe}_{2}$ heterobilayers. Nat. Mater. 19, 617-623 (2020).

57. Zollner, K., Junior, P. E. F. \& Fabian, J. Strain-tunable orbital, spin-orbit, and optical properties of monolayer transition-metal dichalcogenides. Phys. Rev. B 100, 195126 (2019).

58. Stahn, J., Pietsch, U., Blaha, P. \& Schwarz, K. Electric-field-induced charge-density variations in covalently bonded binary compounds. Phys. Rev. B - Condens. Matter Mater. Phys. 63, $165205(2001)$. 


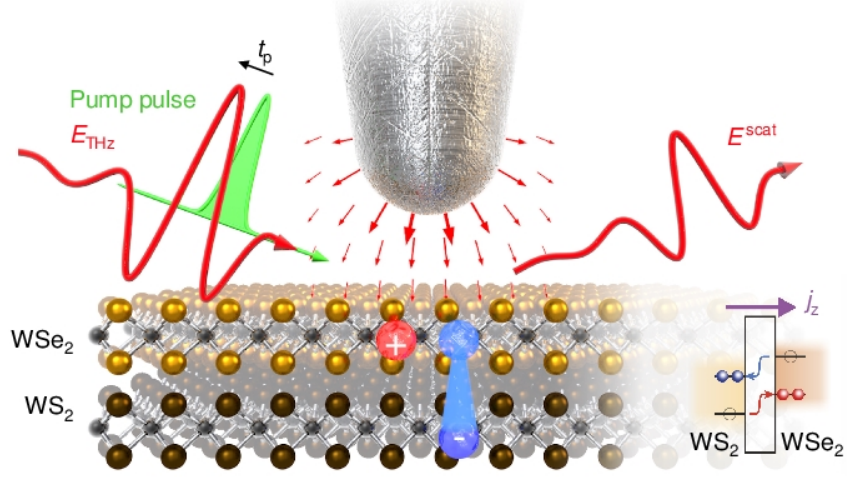

b

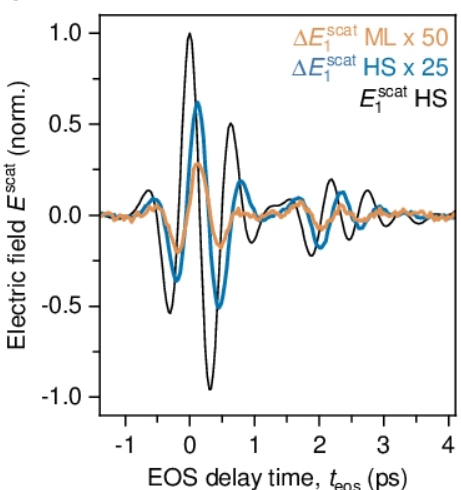

C
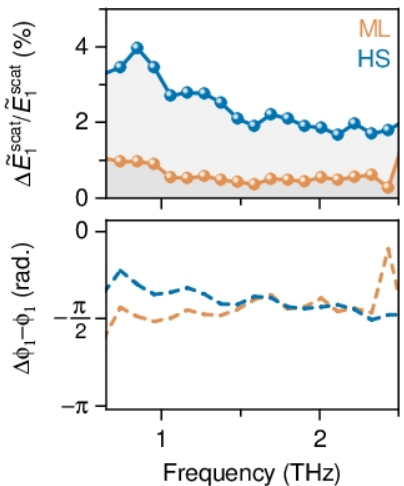

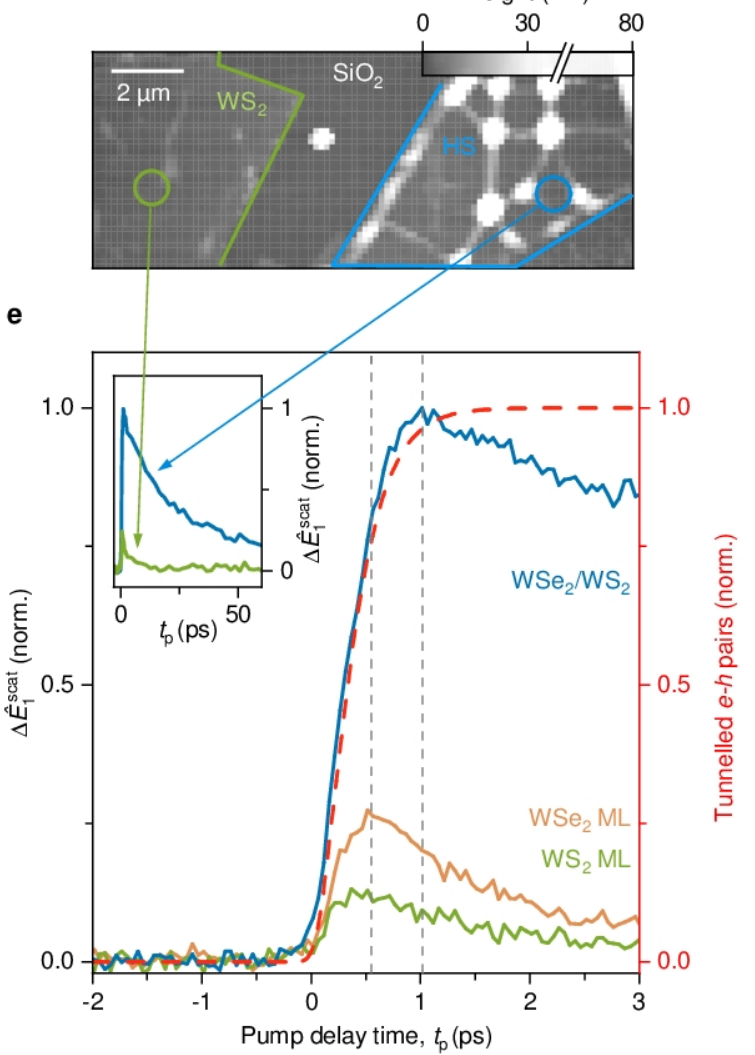



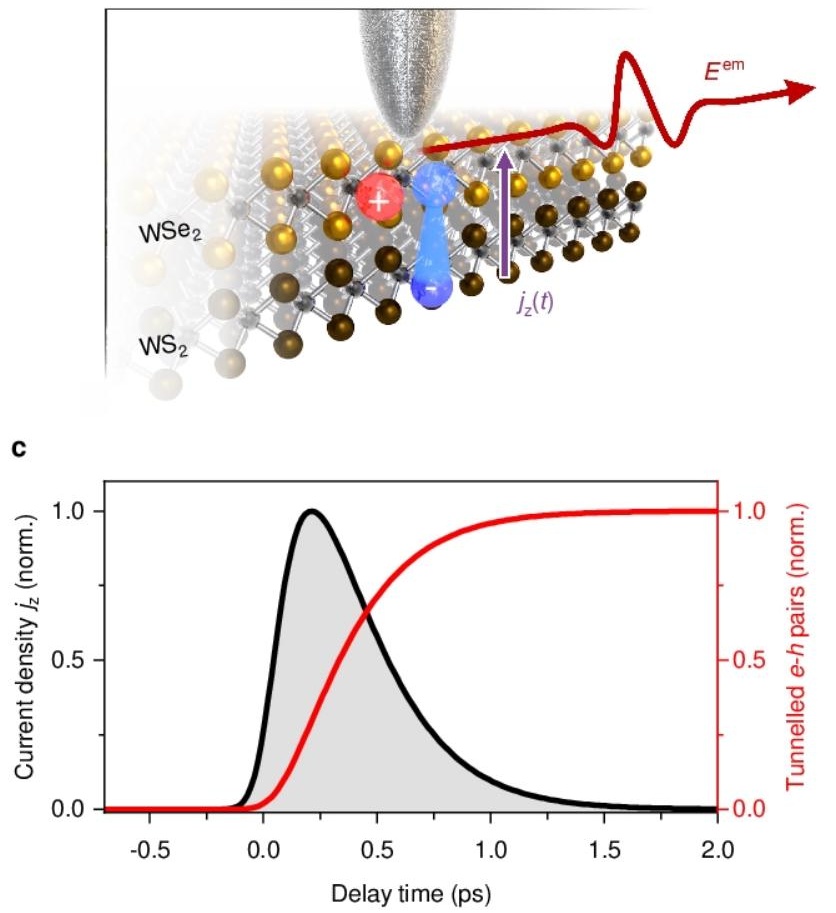

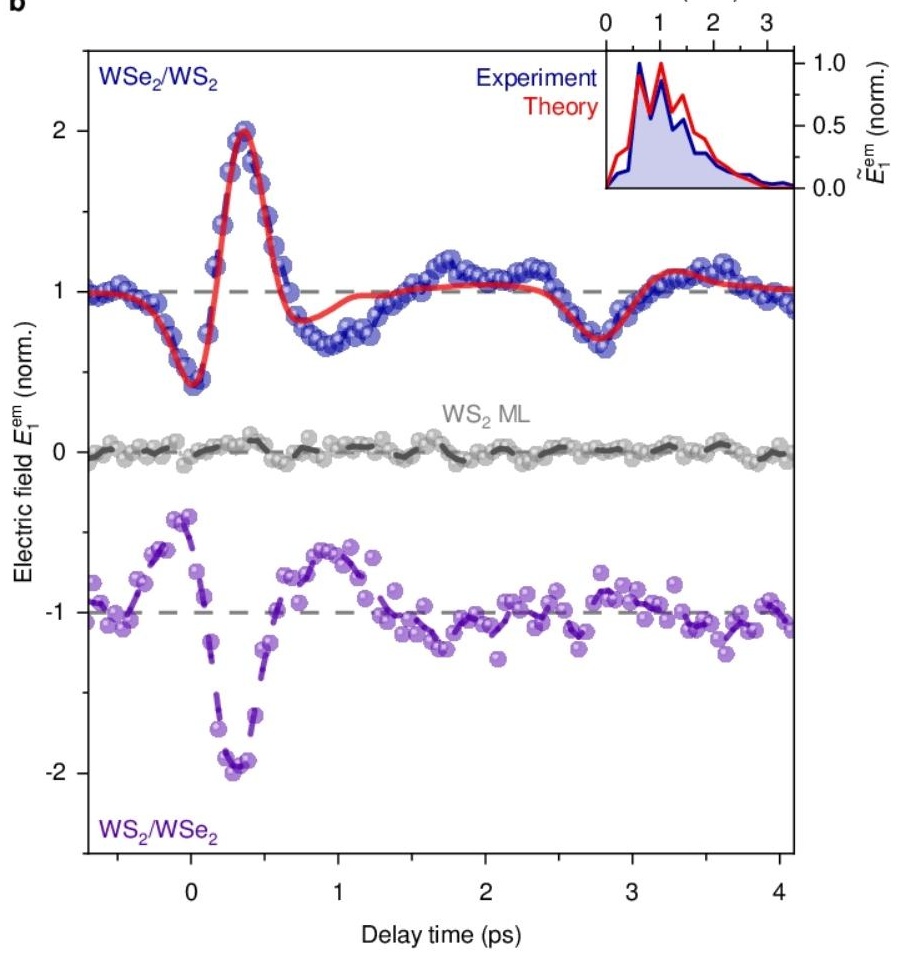



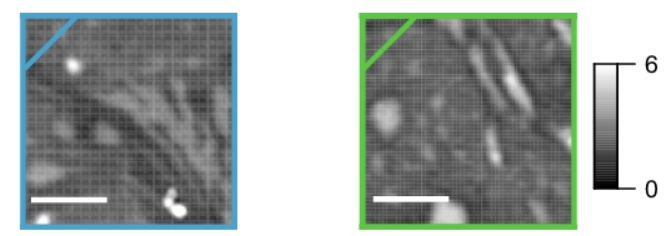

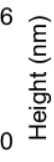

0

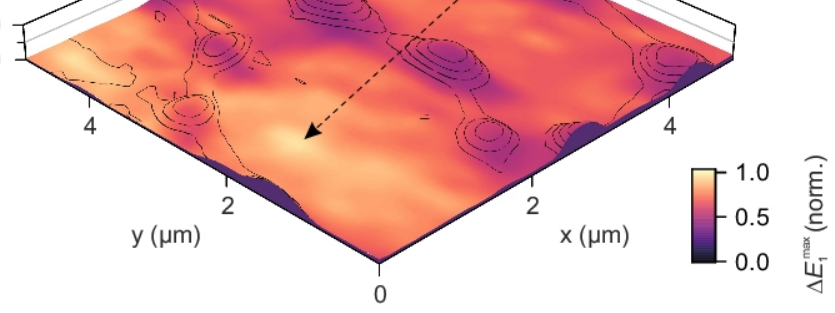

b

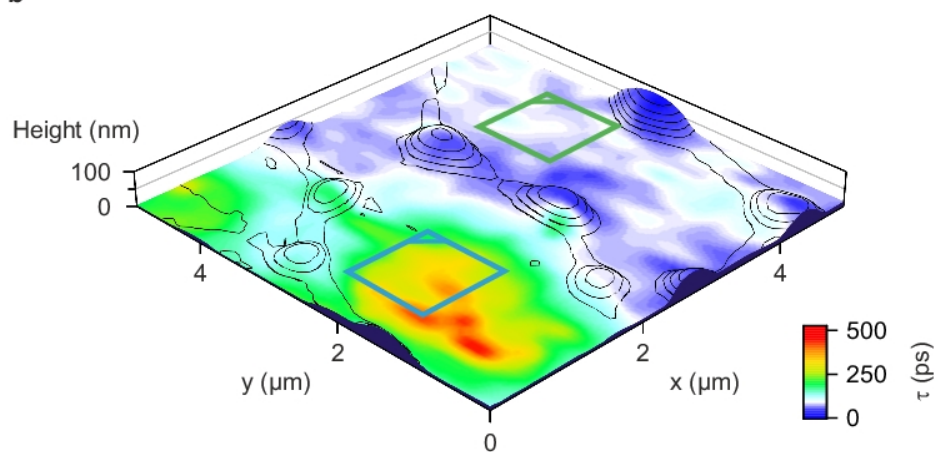

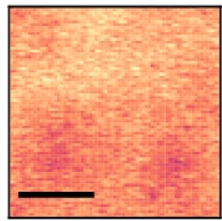

$t_{\mathrm{p}}$

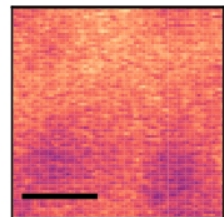

1 ps
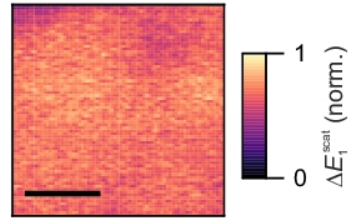

$41 \mathrm{ps}$

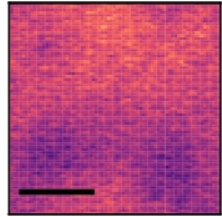

101 ps

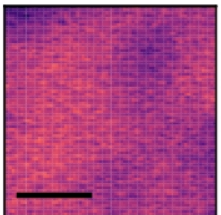

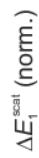

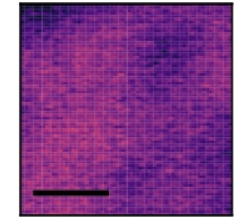

